\title{
Systematic tendencies in scene viewing
}

\author{
Benjamin W. Tatler \\ University of Dundee
}

\author{
Benjamin T. Vincent \\ University of Dundee
}

\begin{abstract}
While many current models of scene perception debate the relative roles of low- and highlevel factors in eye guidance, systematic tendencies in how the eyes move may be informative. We consider how each saccade and fixation is influenced by that which preceded or followed it, during free inspection of images of natural scenes. We find evidence to suggest periods of localized scanning separated by 'global' relocations to new regions of the scene. We also find evidence to support the existence of small amplitude 'corrective' saccades in natural image viewing. Our data reveal statistical dependencies between successive eye movements, which may be informative in furthering our understanding of eye guidance.
\end{abstract}

\section{Keywords: Saccade, fixation, eye guidance, local, global, corrective saccade}

\section{Introduction}

All visually guided behaviours require that we sample relevant information from the visual environment. Because we sample the visual environment using the variable resolution retinae the locations that are selected as targets for the high-resolution fovea are extremely important; they are a fundamental selection bottleneck in extracting and thus processing information from the world. Therefore, it is not surprising that since the selectivity of the oculomotor system was first described, a great deal of research effort has been devoted to trying to understand the factors that might underlie these selection mechanisms (since Buswell, 1935; see Wade \& Tatler, 2005).

Since behaviour is an ongoing process we are interested in describing the moment-to-moment relocations of gaze that allow for successful completion of these behaviours. The research challenge therefore is to characterise the factors that underlie the moment-to-moment decisions about where to direct the eyes each time we make a saccade. To date the majority of research into factors affecting saccade targeting have focused on the roles of lowlevel visual feature cues or high-level factors such as object- or semantic-level cues, or behavioral task demands.

\section{Low-level factors in scene viewing}

Visual search experiments have shown clear and robust influences of various manipulations of basic visual features on the deployment of visual attention. Correspondingly, basic visual features have been given a prominent role in many models of visual attention, such as feature integration theory (Treisman \& Gelade, 1980), guided search (Wolfe, 1998), and attentional engagement theory (Duncan \& Humpreys, 1989, 1992). Recent years have seen the extension of this approach, suggesting that such basic visual features, combined in particular ways, play a fundamental role in guiding attention in natural viewing situations while completing behaviors. One prominent account suggests that eye guidance in these situations can be explained by the local contrast, or salience of sets of features (Itti \& Koch, 2000).

A common approach for evaluating the influence of visual features in targeting saccades has been to attempt to find differences between visual features at the locations that are fixated and at appropriately selected control locations (e.g., Mannan, Ruddock \& Wooding, 1997; Reinagel \& Zador, 1999; Krieger, Rentschler, Hauske, Schill \& Zetzsche, 2000; Parkhurst, Law \& Niebur, 2002; Parkhurst \& Niebur 2003; Tatler, Baddeley \& Gilchrist, 2005; Baddeley \& Tatler 2006; Tatler, Baddeley \& Vincent, 2006). The assumption is that the dimensions in which fixated locations differ from control locations are 
good candidates for visual features that are selected as targets for the eyes to fixate. If the fixated regions contain more of the feature than would be expected from random sampling, then it can be suggested that there was nonrandom selection of this feature by the eyes. Typically such studies show robust differences between fixated and control locations and, particularly, that the greatest differences occur for luminance contrast and edge information (e.g., Parkhurst et al., 2002; Tatler et al., 2005). Baddeley and Tatler (2006) used an approach that employed Bayesian inference explicitly to derive the visual features guiding the eye and found that edges, rather than luminance contrast, were the strongest predictors of fixation locations.

While these findings of higher-than-expected visual features at fixation have been used to support the notion of salience as a causal factor in fixation selection, others have suggested that caution should be exercised when interpreting a correlation between image features and fixation selection as causal (e.g., Henderson, Brockmole, Castelhano \& Mack, 2007). In the present issue of JEMR, Nyström and Holmqvist (2008) offer a clear demonstration that the correlation may be artefactual: Semantically meaningful stimuli can often co-occur with low-level 'salience'. However when 'salience' is removed by manipulating contrast at semantically meaningful locations, observers still fixate these locations. Tatler (2007) explored the relationship between the overall distribution of basic visual features (brightness, chromaticity, contrast and edges) in scenes and the overall fixation distributions of observers viewing these scenes. This study found little or no correlation between simple features and fixation distributions and thus demonstrated the lack of a causal link between the distribution of low-level visual features and fixations. Of course this result should not be taken to suggest that eye guidance is not in any way influenced by the visual information present. Instead we, like others, take this result to suggest that a more prominent role is played by high-level visual and behavioural factors.

\section{High-level factors in scene viewing}

A strong challenge to the feature-driven accounts of eye movement behaviour comes from the finding that where we look is strongly influenced by the behavioural goals of the observer. Early demonstrations of this by Buswell (1935) and Yarbus (1967) showed that when observers view the same scene but with different instructions, the resultant patterns of fixation differ greatly. It is clear in natural behaviour that where we look is tightly coupled to the task we are undertaking (e.g., Ballard et al., 1992; Ballard, Hayhoe \& Pelz, 1995) and we rarely look at objects or locations that are not relevant to the current task (e.g., Hayhoe, Shrivastava, Mruczek \& Pelz, 2003; Land \& Hayhoe, 2001; Land, Mennie \& Rusted, 1999). Therefore, for natural viewing behaviour, while basic visual features may play a minor role, visual information is of course utilized, but at a higher, object- or sematic-level. This notion is supported by the finding that the correlation between salience (as assessed using the Itti and Koch, 2000, algorithm) and fixations, varies with task. The salience model provides a more effective account of free viewing behaviour than search behaviour (Underwood \& Foulsham, 2006; Underwood, Foulsham, van Loon, Humphreys \& Bloyce, 2006). A number of recent studies have also supported the notion that behavioural task overrides any correlation between visual salience and fixation (Einhäuser, Rutishauser, \& Koch, 2008; Henderson et al., 2007).

One attempt to improve feature-based accounts for targeted searches has been to suggest that high-level aspects of behaviour can be captured by selectively weighting the various feature channels (Navalpakkam and Itti, 2005). Here, each feature channel (edges, colour, etc.) can be weighted so as to hopefully result in a target object having highest salience. This extension to the account, which can be thought of as top-down knowledge of the feature properties of a target object, is certainly a step in the right direction in terms of accounting for taskbased effects. However when evaluated with natural images and incorporating variable retinal resolution the ability for a model to fixate targets is still limited (Vincent, Troscianko \& Gilchrist, 2007).

Another successful extension to a feature-based approach is to consider the role of high-level scene gist, combined with prior knowledge of where objects are typically located (Torralba, 2003; Torralba, Oliva, Castelhano \& Henderson, 2006). These authors suggest that when we are presented with a scene, we rapidly extract the overall gist of the scene and use this to generate a set of spatial prior probability distributions that describe where particular objects are likely to be found. These spatial priors can be used to modulate feature-based information so as to constrain search to plausible target locations. Empirical testing of this idea has shown that a model that incorporates both the visual characteristics of 
the scene and the likely location of targets is much better able to account for human oculomotor behaviour, than a model based on the visual characteristics alone (Torralba et al., 2006).

\section{Systematic tendencies in scene viewing}

Despite considerable research effort to model and understand the contribution of low- and high-level factors in eye guidance, our ability to accurately model and predict eye movement behaviour remains relatively limited. In contrast, there have recently been significant advances in our ability to model other types of action selection and motor behaviour successfully (Körding \& Wolpert, 2004). It is now recognized that action selection is heavily influenced by the fact that motor behaviours are not all equally likely to be selected: For example, systematic biases to make certain combinations of finger movements over others are highly informative in our understanding of natural behaviour of the hand (Ingram, Körding, Howard, \& Wolpert, 2008). Equivalent approaches have yet to be applied to understanding oculomotor behaviour and the extent to which these factors play a role in eye guidance is the focus of current work in our research group (Tatler \& Vincent, in preparation). However, we feel that there is ample evidence from existing literature that systematic tendencies in oculomotor behaviour exist.

Systematic tendencies in oculomotor behaviour can be thought of as regularities that are common across all instances of and manipulations to the behaviour. Such tendencies can be seen in saccade amplitudes, which show a positively skewed, long-tailed distribution in most experimental settings in which complex scenes are viewed (e.g., Bahill, Adler \& Stark, 1975; Gajewski, Pearson, Mack, Bartlett \& Henderson, 2005; Pelz \& Canosa, 2001; Tatler et al., 2006). Saccade directions have also been shown to be non-uniformly distributed (e.g., Bair \& O’Keefe, 1998; Lappe, Pekel \& Hoffmann, 1998; Lee, Badler \& Badler, 2002; Moeller, Kayser, Knecht \& König, 2004) with saccades more frequently initiated in the horizontal and vertical directions than in oblique directions.

Not only have these 'first order' tendencies been observed, but also, more complex dependencies between successive saccades and fixations are evident. Motter and Belky (1998) found that there were sequential dependencies in saccade amplitudes, with small amplitude saccades tending to be followed by long amplitude saccades and vice versa. A relationship between fixation duration and the amplitude of the saccade that follows it has previously been reported (Unema, Panasch, Joos \& Velichkovsky, 2005; Velichkovsky, Rothert, Kopf, Dornhoefer, \& Joos, 2002; Velichkovsky, Rothert, Miniotas, Dornhoefer, Joos, \& Pannasch, 2003), but other studies provide evidence both for and against such a relationship (e.g., Pelz \& Canosa, 2001; Viviani, 1990; Viviani \& Swensson, 1982). Hooge, Over, van Wezel and Frens (2005) found that saccades are frequently in the same direction as their immediate predecessor. These authors also looked at change in amplitude between successive fixations and found that the magnitude of this change tended to fall within 10 degrees.

While reports of sequential dependencies between successive saccades and fixations therefore can be found in the literature, a systematic exploration of how each saccade and fixation may relate to each other has yet to be conducted. If we are to understand the nature of any sequential tendencies in looking behaviour, a systematic exploration of the possible dependencies is required. This is the first aim of the present report: to explore where reliable tendencies between saccades and fixations are found.

Moreover, two questions about how we look at scenes emerge from this consideration:

1) In simple lab-based viewing paradigms it is often observed that large amplitude saccades fall short of the intended target (the are hypometric) and are followed by a small amplitude corrective saccade (e.g., Becker, 1972, 1991). Whether a systematic tendency to make small amplitude corrective saccades after large amplitude saccades is a feature of viewing natural scenes remains to be determined, and is the second issue that will be considered throughout the current report.

2) There is a suggestion that there may be at least two qualitatively distinct modes of viewing scenes: global and local scanning (e.g., Frost \& Pöppel, 1976; Unema et al., 2005; Velichkovsky, Joos, Helmert, \& Pannasch, 2005; Velichkovsky et al., 2002, 2003). Global scanning periods are associated with large amplitude saccades and short duration fixations, whereas local scanning periods are associated with smaller amplitude saccades and longer fixations. In general it is argued that global scanning is restricted to the first two seconds of viewing photographic scenes (Unema et al., 2005; Velichkovsky et 
al., 2005) For a thorough exploration of these two viewing modes see Pannasch, Helmert, Roth, Herbold and Walter (2008). However, from natural behaviour studies we see evidence for periodic large amplitude relocations to new regions, followed by small amplitude saccades exploring the new region (Land, Mennie \& Rusted, 1999). It may therefore be that global relocations are not a feature of only the first two seconds of viewing but are interspersed throughout viewing. It is this latter issue that we will also consider over the course of the analyses presented in this paper.

While an exploration of the possible systematic tendencies in how we move our eyes is informative and not previously available from the literature, it is important to consider how any evidence we find for systematic tendencies might bear on existing understanding and models of eye guidance. At present models do not recognize the existence of any systematic component of how we move our eyes that might be independent of low-level image features and high-level aspects of the present behaviour. We do not suggest that finding systematic tendencies in how we move our eyes should argue against low- or highlevel factors in scene viewing; however, if such tendencies are found then these offer a previously-untapped component for future models of eye guidance. As already stated, we have seen in recent work in other motor domains that incorporating systematic tendencies in hand behaviour dramatically improves the ability to model hand movements. Such a benefit for oculomotor models may also be offered by understanding systematic tendencies.

In the current paper we present an exploration of systematic tendencies between fixation durations, saccade amplitudes and saccade directions of successive eye movements. This exploration is designed to serve as a launch point for future research, highlighting where sequential dependencies exist and therefore from where useful information about how the observer will move their eyes can be found. How informative these reported dependencies are in eye guidance is beyond the scope of the present report but will be an interesting avenue for future research.

\section{Method}

\section{Participants}

Twenty-two participants (aged 18-29; 19 male) took part in the experiment. All had normal or corrected-tonormal vision and were naïve to the purposes of the experiment. Participants received either course credit or monetary reward for taking part in the experiment.

\section{Stimuli and procedure}

Participants each viewed 120 photographs of natural scenes: 40 indoor, and 80 outdoor scenes (40 with man made structures contained in them, 40 with no man made structures visible). Images were taken using a Nikon D2 digital SLR using the highest resolution (4 megapixels).

Images were displayed in $1600 \times 1200$ pixel format on a 21 " SVGA colour monitor with a refresh rate of 100 Hz. Viewers were positioned $60 \mathrm{~cm}$ from the monitor such that the images subtended $40^{\circ}$ horizontally and $30^{\circ}$ vertically of the observer's field of view.

Participants were given no specific task instructions, merely being asked to freely view the images. Before each image was presented, participants were required to fixate a small marker positioned randomly within $10^{\circ}$ of the centre of the screen. Images were presented for five seconds and were followed by a white noise mask.

\section{Eye movement recording}

Eye movements were recorded using an SR Research Ltd. EyeLink II eye tracker, sampling pupil position at $500 \mathrm{~Hz}$. The eye tracker was calibrated using a 9-point grid. A second 9-point grid was used to calculate the accuracy of the calibration; if the spatial accuracy on the second 9-point grid was worse that $\pm 0.5^{\circ}$, the eye tracker was re-calibrated. Eye position data were collected for the eye that produced the better spatial accuracy as determined using the calibration. Saccades and fixations were defined using the saccade detection algorithm supplied by SR Research: Saccades were identified by deflections in eye position in excess of $0.1^{\circ}$, with a minimum velocity of $30^{\circ} \mathrm{s}^{-1}$ and a minimum acceleration of $8000^{\circ} \mathrm{s}^{-2}$, maintained for at least $4 \mathrm{~ms}$. We employed a minimum fixation duration of $50 \mathrm{~ms}$. The first fixation in each trial was defined as the first fixation that began after the onset of the scene image. Thus the fixation on the pretrial fixation marker was not included in the analyses. 
After all exclusions, 40,905 fixations (and saccades) were available for subsequent analyses.

\section{Analysis}

We explored how each fixation and saccade is influenced by its predecessor and successor. Figure 1 shows a schematic of a sequence of eye movements in order to illustrate the quantities that we extracted and analysed. We thus extracted the current fixation duration $\left(\mathrm{FD}_{\mathrm{N}}\right)$ and previous fixation duration $\left(\mathrm{FD}_{\mathrm{N}-1}\right)$; the current saccade amplitude $\left(\mathrm{SA}_{\mathrm{N}}\right)$ and previous saccade amplitude $\left(\mathrm{SA}_{\mathrm{N}-1}\right)$; and the current saccade direction $\left(\mathrm{SD}_{\mathrm{N}}\right)$ and previous saccade direction $\left(\mathrm{SD}_{\mathrm{N}-1}\right)$. This diagram will be used throughout the Results and Discussion section to clarify which parings of eye movement measures are under exploration in each part of the analyses.

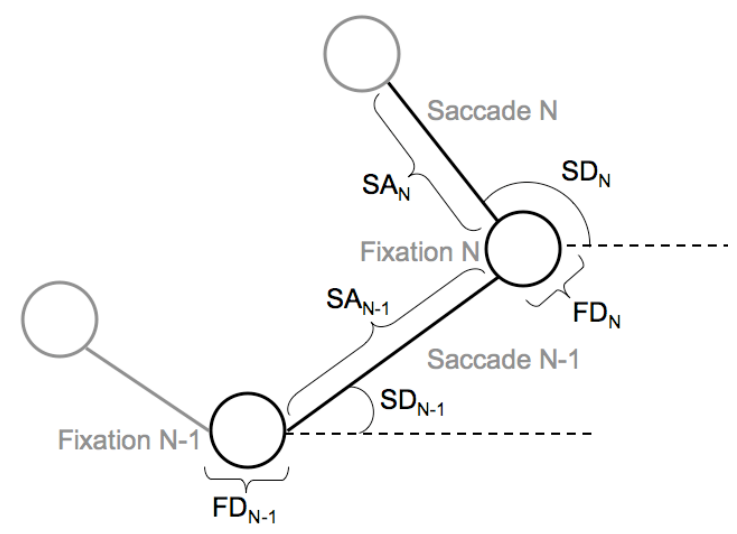

Figure 1. Schematic of a sequence of four fixations, denoted by the circles, with lines representing the three saccades that separated these fixations. The black fixations and saccades are those explored in the present study. The measured characteristics of these saccades and fixations are also shown for fixation duration (FD), saccade amplitude (SA) and saccade direction (SD) for each of the current (N) and previous (N-I) saccade or fixation.

In initial characterizations of the relationships between each combination of these variables, we plotted the joint distribution, essentially a density plot showing how probable any given combination of two variables is. For the few cases that showed clear multimodality, we present this joint distribution density plot. However, when the relationship between variables was unimodal, we calculate both conditional distributions. These show how probable one variable (A) is given that we already know the value of the other (B). It is important to realize
$\mathrm{P}(\mathrm{A} \mid \mathrm{B})$ does not necessarily equal $\mathrm{P}(\mathrm{B} \mid \mathrm{A})$. For these conditional distributions we plot the median values with $95 \%$ confidence intervals of the median, estimated from 1000 bootstrapped samples (see Efron and Tibshirani, 1993). Given a Gaussian distribution and no outliers, then the mean is the best measure of central tendency, however for much of the data we graph, the distributions are both skewed and have outliers, therefore we chose to use the median as a measure of central tendency. Bootstrap is a resampling procedure which is a very useful technique to calculate the confidence, in this case of the actual median values.

\section{Results and discussion}

In this section we explore systematic tendencies in scene viewing by considering what factors influence the duration of fixations and the amplitude and direction of saccades while viewing images of natural scenes.

\section{Fixation duration}

Let us first consider the relationship between the durations of two successive fixations depicted as a joint probability distribution in Figure 2A. This joint distribution can be characterised by two conditional distributions: the median current fixation duration given a particular previous fixation duration (Figure 2C), and the median previous fixation given a particular current fixation duration (Figure 2D). Note that these conditional distributions can, and here do, show very different dependencies between the two variables. Figure $2 \mathrm{C}$ shows that longer fixations tend to be followed by longer fixations, which could reflect a frequently-observed general tendency to increase fixation duration over the course of several seconds of viewing (e.g., Antes, 1974; Buswell, 1935; Unema et al., 2005). Figure 2D shows a non-linear relationship between the current fixation duration and the median of the preceding fixation: There is a U-shaped function where fixations lasting around 100-200 ms tend to have been preceded by short duration fixations, whereas those lasting either less ${ }^{1}$ or more time than this tend to have been preceded by long fixations.

\footnotetext{
${ }^{1}$ While we employed a minimum fixation duration criterion of $50 \mathrm{~ms}$, we are still able to consider fixations lasting less than $100 \mathrm{~ms}$ in our analyses. Any limitations in the number of samples in this range are reflected in the bootstrapped error bars.
} 
A.

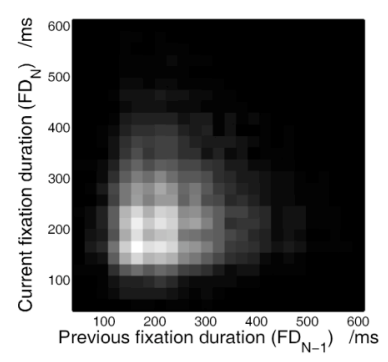

B.

C.
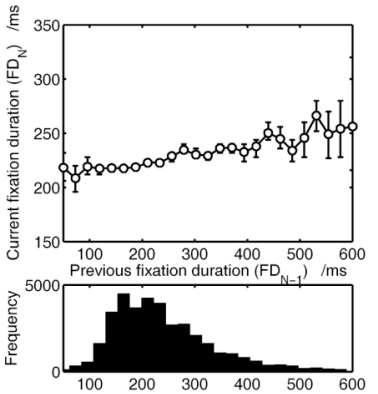

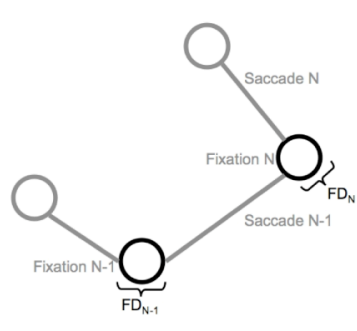

D.

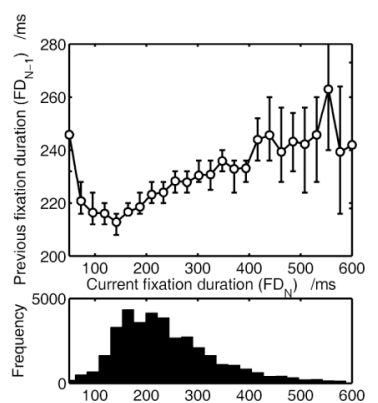

Figure 2. (A) The joint distribution showing the relationship between current fixation duration $\left(F D_{N}\right)$ and previous fixation duration $\left(F D_{N-1}\right)$. (B) Diagram illustrating the two quantities under investigation in this Figure: the fixation durations of two successive fixations. (C) Median current fixation duration (with 95\% confidence intervals) as a function of previous fixation duration. The histogram in the lower panel of this plot shows the data that contribute to each bin in the upper panel. (D) Median previous fixation duration (with $95 \%$ confidence intervals) as a function of current fixation duration. The histogram in the lower panel of this plot shows the data that contribute to each bin in the upper panel.

\section{Saccade amplitude}

Figure 3 explores the relationship between the amplitudes of successive fixations. There is a strong non-linear relationship between current and previous saccade amplitudes: Very small amplitude saccades tend to have been preceded by small amplitude saccades (Figure 3D). There is a positive relationship between current and previous saccade amplitude as the current amplitude increases to about 3 degrees. Following this, there is a gradual trend toward larger amplitude saccades tending to have been preceded by smaller saccades. Our finding that small amplitude saccades tend to have been preceded by other small amplitude saccades suggests that there may occur sequences of short amplitude saccades in the eye movement records of our observers. This relationship is not the

same as that reported by Motter and Belky (1998), who found no evidence for sequences of small amplitude fixations and instead suggested that small saccades tended to be followed by longer saccades and longer saccades tended to be followed by short ones. It should be remembered, however, that Motter and Belky (1998) used simple search arrays rather than complex photographic scenes. In these search arrays, targets were small and uniformly sized and there were necessarily large portions

of the screen that contained no visual targets. Thus the amplitudes of saccades executed are likely to be closely related to the spacing between targets. In contrast, photographic scenes contain continuous visual textures and objects of varying sizes, placed in less ordered arrangements.
A.

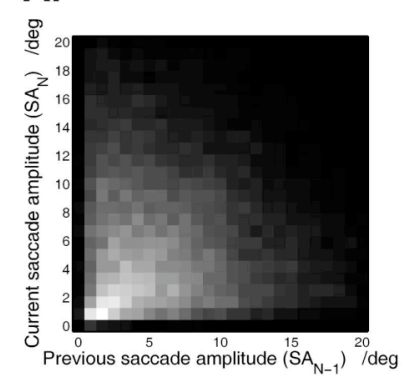

C.

疍

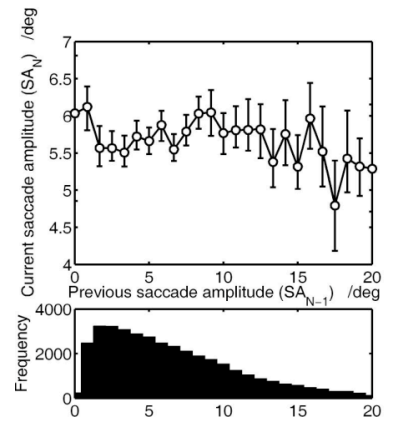

B.

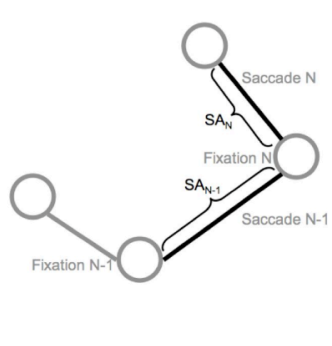

D.

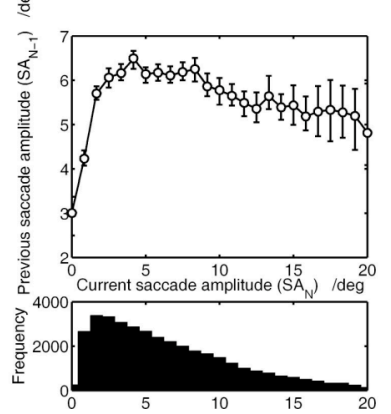

Figure 3. (A) The joint distribution showing the relationship between current saccade amplitude $\left(S A_{N}\right)$ and previous saccade amplitude $\left(S A_{N-1}\right)$. (B) Diagram illustrating the two quantities under investigation in this Figure: the amplitudes of two successive saccade. (C) Median current saccade amplitude (with $95 \%$ confidence intervals) as a function of previous saccade amplitude. The histogram in the lower panel of this plot shows the data that contribute to each bin in the upper panel. (D) Median previous saccade amplitude (with $95 \%$ confidence intervals) as a function of current saccade amplitude. The histogram in the lower panel of this plot shows the data that contribute to each bin in the upper panel. 
Our suggestion that there may occur sequences (or at least repetitions) of small amplitude saccades can be used to re-visit previous suggestions that there may be at least two qualitatively distinct 'modes' of viewing complex scenes - a local scanning strategy, characterised by small amplitude saccades and selection of conspicuous visual features, and a more global strategy, characterised by large amplitude saccades to regions that are not visually conspicuous in terms of their feature content (e.g., Frost \& Pöppel, 1976; Tatler et al., 2006). It has been suggested that global processing may be restricted to the first two seconds of viewing (Pannasch et al., 2008; Unema et al., 2005) but as we argued in the Introduction, evidence for natural behaviour suggests that global relocations to new scene regions may persist throughout tasks. In our analyses we do not consider differences early and later in scene viewing, but consider whether there is any evidence for periods of global and local scanning over the entire viewing period.

We therefore expected to find evidence for sequences of small amplitude saccades and this is shown in Figure 3D. However, we find no strong evidence for sequences of large amplitude saccades ${ }^{2}$. Instead we find that large amplitude saccades (in excess of about 8 degrees) tend to have been preceded by smaller amplitude saccades. This pattern would be consistent with a viewing strategy in which large saccades to new regions of a scene tend to follow small locally-explorative saccades, but are then themselves followed by more local exploration of the new scene region, rather than a series of larger saccades.

\section{Saccade direction}

Figure 4 shows the relationship between the directions of consecutive saccades ${ }^{3}$. To see the individual hisograms for the directions of the previous and current saccades, see the plots in the lower panels of Figure $8 \mathrm{E}$ and $\mathrm{F}$. These show that saccades are most common in the horizontal direction, followed by vertical directions.

\footnotetext{
2 The lack of support for sequences of large amplitude saccades might be taken as evidence against a period of global scanning comprising large amplitude saccade at the start of viewing. However, without breaking down the viewing period into early and late, we cannot make strong claims about this.

${ }^{3}$ The multimodality of distributions of both previous and current saccade directions means that it is not meaningful to plot the conditionals of this joint distribution.
}

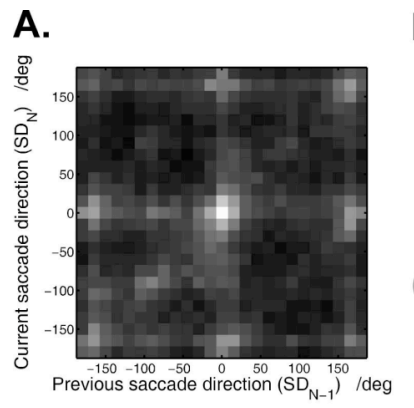

B.

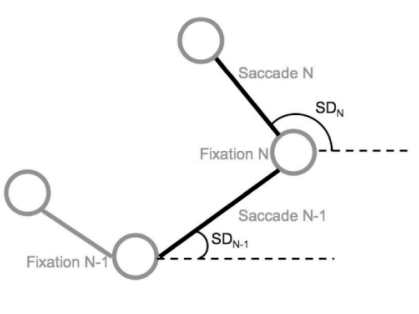

Figure 4. (A) The relationship between current saccade direction $\left(S D_{N}\right)$ and previous saccade direction $\left(S D_{N-1}\right)$. Conditionals are not plotted as the distributions of saccade direction are multimodal. Note that negative values represent saccades directed in upward directions, positive values denote saccades directed in downward directions. Zero is a rightward saccade, 180 is a leftward saccade. (B) Diagram illustrating the two quantities under investigation in this Figure: the directions of two successive saccades.

Motter and Belky (1998) found little evidence for sequential dependencies in the directions of successive saccades, apart from a tendency to initiate a saccade in the opposite direction and of the same amplitude as the previous saccade - i.e. to return to the previous fixation location. They otherwise concluded that each saccade was essentially randomly directed with respect to the previous. In our data, for participants viewing complex scenes rather than search arrays, we find that there is an overall bias to make saccades either in the same direction as the previous saccade, or that are 180 degree reversals The pattern to either continue or reverse saccade direction is hard to explain within the context of natural scene viewing. However, this pattern is consistent with the possibility that there is some degree of 'inaccuracy' of saccade targeting followed by a 'corrective' saccade to fixate the target of interest: hypometric (i.e. undershoot) saccades would be followed by saccades in the same direction to bring the fovea to bear on the intended target; hypermetric (i.e. overshoot) saccades would require a saccade in the opposite direction to fixate the intended target. While there is ample evidence for corrective saccades when viewing simple stimuli (e.g., Becker, 1972, 1991; Carpenter, 1988; Deubel, Wolf \& Hauske, 1982; Kapoula \& Robinson, 1986; Prablanc \& Jeannerod, 1975), whether corrective saccades are a feature of complex scene viewing or activities in real environments remains unclear (e.g., Ballard et al., 1992; Hayhoe et al., 2003; Land \& Hayhoe, 2001; Pelz \& Canosa, 2001). We shall return to 
the possibility of 'corrective' saccades in our dataset later.

\section{Fixation duration and saccade amplitude}

Previous studies have debated whether or not there exists a strong relationship between fixation duration and the amplitude of the saccade that immediately follows the fixation when viewing scenes (e.g., Pelz \& Canosa, 2001; Viviani, 1990; Viviani \& Swensson, 1982). In this section we consider not only the relationship between fixation duration and the amplitude of the following saccade, but also the relationship between fixation duration and the amplitude of the preceding saccade.
A.

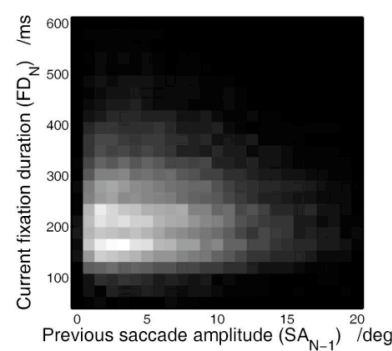

c.

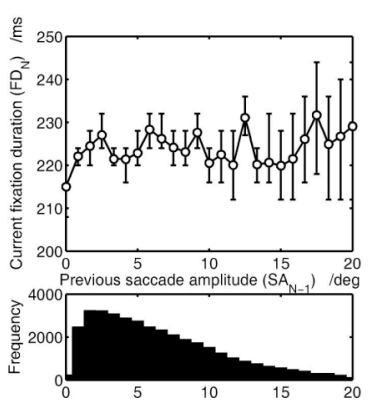

B.

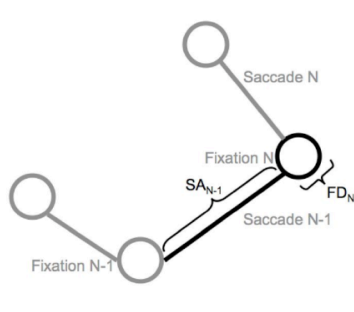

D.

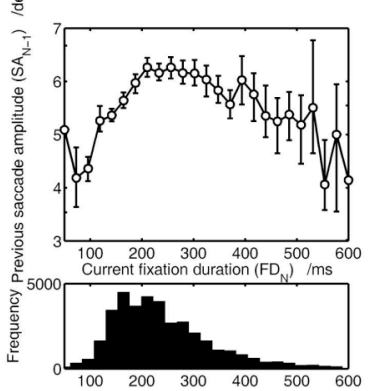

Figure 5. (A) The joint distribution showing the relationship between current fixation duration $\left(F D_{N}\right)$ and previous saccade amplitude $\left(S A_{N-1}\right)$. (B) Diagram illustrating the two quantities under investigation in this Figure: the duration of a fixation and the amplitude of the saccade that immediately preceded it. (C) Median current fixation duration (with 95\% confidence intervals) as a function of previous saccade amplitude. The histogram in the lower panel of this plot shows the data that contribute to each bin in the upper panel. (D) Median previous saccade amplitude (with $95 \%$ confidence intervals) as a function of current fixation duration. The histogram in the lower panel of this plot shows the data that contribute to each bin in the upper panel.

The relationship between fixation duration and the amplitude of the saccade that immediately preceded that fixation is shown in the joint distribution depicted in Figure 5A. The conditional medians (Figure 5C,D) show very different trends: Knowing the amplitude of the saccade does not allow us to predict the duration of the following fixation (Figure 5C). However, we can use the duration of the fixation to characterise the saccade that brought the eye to this location (Figure 5D). Here we see that the frequent intermediate duration fixations (about 200-300 $\mathrm{ms}$ in duration) tend to have been preceded by larger amplitude saccades than either very short or very long duration fixations.
A.

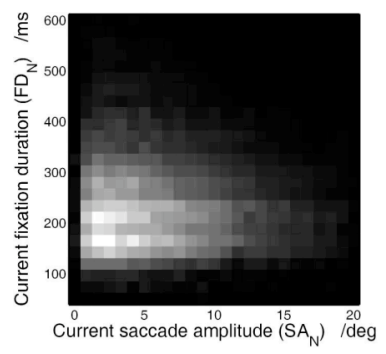

C.

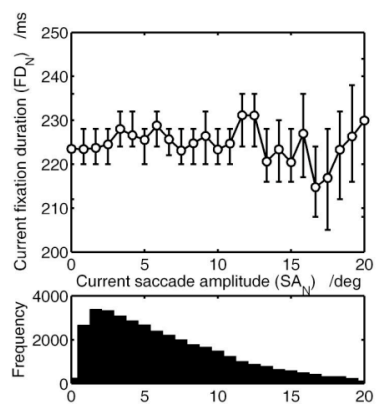

B.

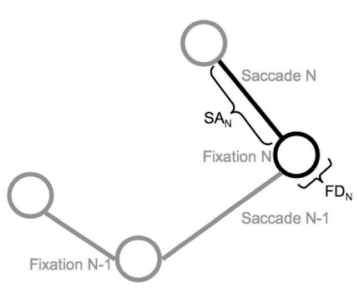

D.

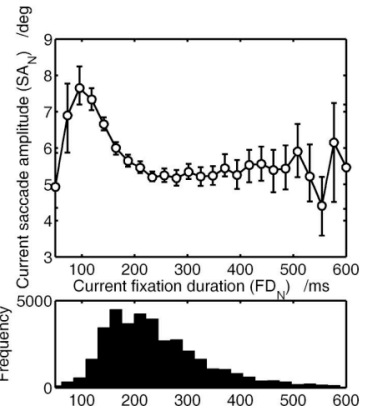

Figure 6. (A) The joint distribution showing the relationship between current fixation duration $\left(F D_{N}\right)$ and current saccade amplitude $\left(S A_{N}\right)$. (B) Diagram illustrating the two quantities under investigation in this Figure: the duration of a fixation and the amplitude of the saccade that immediately followed it. (C) Median current fixation duration (with $95 \%$ confidence intervals) as a function of current saccade amplitude. The histogram in the lower panel of this plot shows the data that contribute to each bin in the upper panel. (D) Median current saccade amplitude (with 95\% confidence intervals) as a function of current fixation duration. The histogram in the lower panel of this plot shows the data that contribute to each bin in the upper panel.

In characterising the relationship between fixation duration and the amplitude of the following saccade (Figure $6 \mathrm{~A}$ ), we again see very different patterns in the two con- 
ditional distributions (Figure 6C,D). Again, the amplitude of the saccade is not informative about the duration of the fixation that preceded it (Figure 6C). However, fixation duration can be used to describe the probable saccade amplitudes that follow: Here we see a strong, non-linear relationship between fixation duration and the amplitude of the following saccade (Figure 6D). Very short $(<80$ ms) fixations are followed by small amplitude saccades; fixations of $80-180 \mathrm{~ms}$ duration tend to be followed by large amplitude saccades, and longer fixations tend to be followed by smaller amplitude saccades. The form of this relationship is strikingly similar to that previously reported by Velichkovsky and colleagues (Unema et al., 2005; Velichkovsky et al., 2002, 2003). These authors interpreted this finding as possibly resulting from two competing parallel processes in saccade targeting: a race for selecting the next 'salient' location in the scene, and inhibitory spatial selection.

In fully describing how fixation duration and saccade amplitude might influence each other, we can also consider how changes in the amplitudes of saccades before and after a fixation influence its duration (Figure 7A,B), or how changes in fixation durations before and after a saccade influence its amplitude (Figure 7C,D).

Figure 7A shows that if a large amplitude saccade is followed by a smaller amplitude saccade $\left(\mathrm{SA}_{\mathrm{N}}-\mathrm{SA}_{\mathrm{N}-1}\right.$ is negative), then the fixation between these two saccades is likely to be longer than if a small saccade is followed by a larger saccade $\left(\mathrm{SA}_{\mathrm{N}}-\mathrm{SA}_{\mathrm{N}-1}\right.$ is positive).

This relationship is again consistent with periods of local scanning followed by large amplitude relocations to new locations in the scene. In particular the observed finding in Figure 7A could reflect changes associated with switching between periods of local scanning and relocations to new scene regions. We have argued above that there is evidence in our data for sequences of small amplitude saccades and single (or few) large amplitude saccades (Figure 3). As such, we can consider the data in Figure 7A in these terms. A switch from a large to a small amplitude saccade would be consistent with the relocation to a new scene location followed by a period of more localised scanning. A switch from a small to a large amplitude saccade would be consistent with the end of localised scanning, before initiating a saccade to a new region of the scene. Thus we could suggest from the data in Figure 7A that the first fixation in a new scene region is longer than the last fixation in an area that has received localised scanning.
A.

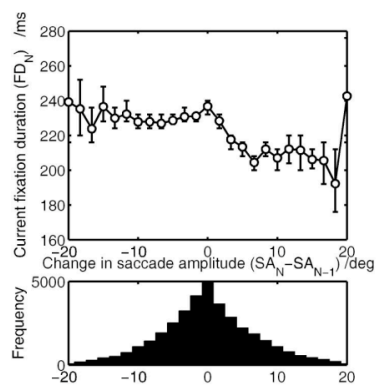

c.

里

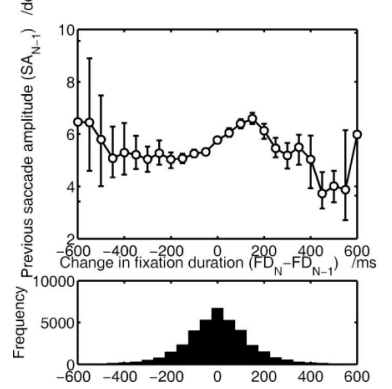

B.

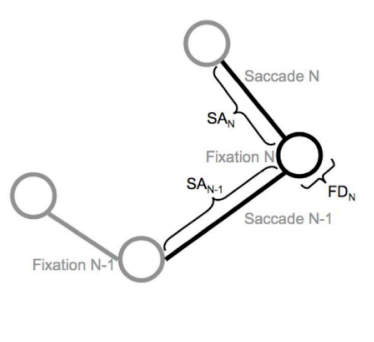

D.

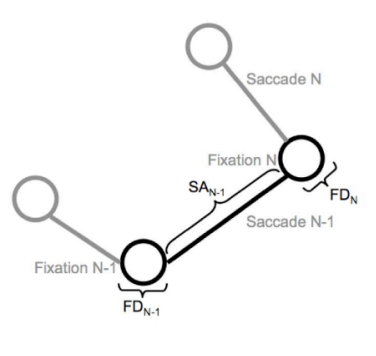

Figure 7. (A) Median current fixation duration (with 95\% confidence intervals) as a function of the absolute change in saccade amplitude between the current and previous saccades. The histogram in the lower panel of this plot shows the data that contribute to each bin in the upper panel. (B) Schematic illustrating the quantities plotted in (A): the duration of a fixation as a function of the change in amplitude between the saccade that followed it and that which preceded it. (C) Median 'previous' saccade amplitude (with 95\% confidence intervals) as a function of the absolute change in fixation duration between the current and previous fixations. The histogram in the lower panel of this plot shows the data that contribute to each bin in the upper panel. (D) Schematic illustrating the quantities plotted in (C): the amplitude of a saccade as a function of the change in duration between the fixation that followed it and that which preceded it.

Figure 7C is consistent with our suggested switching between local and global inspection strategies. Here we see that if a fixation is longer than its predecessor, the saccade between these two fixations tends to be of larger amplitude (for differences of between 0 and $200 \mathrm{~ms}$, which represent with the most frequent positive differences in fixation duration in our data set). Thus a short duration fixation followed by a longer duration fixation will tend to have been separated by a large amplitude saccade. This plot therefore shows the same sequential 
dependencies between saccade amplitude and fixation duration as suggested by Figure 7A.

\section{Fixation duration and saccade direction}

A relatively unexplored aspect of oculomotor behaviour when viewing complex scenes is to explore the possible relationships between fixation duration and saccade direction. Once again we consider the relationship between the duration of a fixation and the direction of the previous or following saccade.
A.

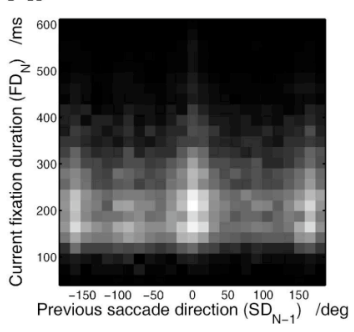

C.

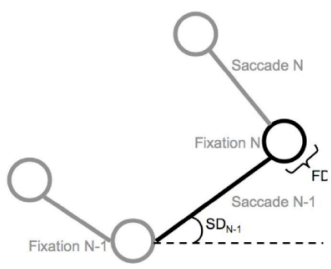

E.

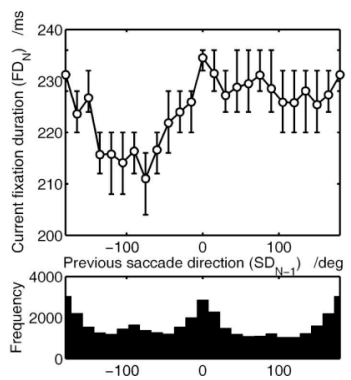

B.

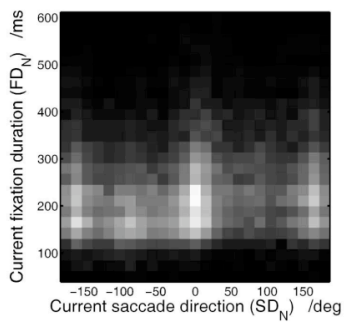

D.

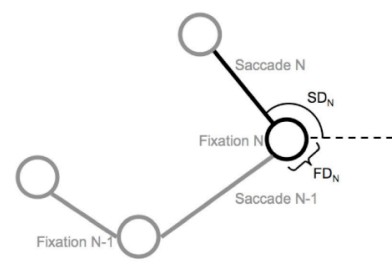

F.

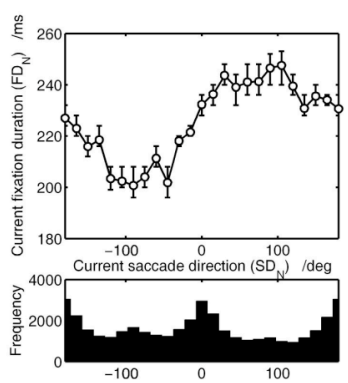

Figure 8. (A,B) Joint distributions showing the relationship between current fixation duration $\left(F D_{N}\right)$ and $(A)$ previous saccade direction $\left(S D_{N-1}\right)$, and $(B)$ current saccade direction $\left(S D_{N}\right)$. Note that negative values represent saccades directed in upward directions, positive values denote saccades directed in downward directions. Zero is a rightward saccade, 180 is a leftward saccade. (C,D) Schematics illustrating the measures plotted in (A) and (C) respectively. (E,F) Median fixation duration (with $95 \%$ confidence intervals) as a function of (E) previous saccade direction, and (F) current saccade direction. The histograms in the lower panels of these plots show the data that contribute to each bin in the upper panels.
Figure 8A,C,E shows the relationship between fixation duration and the direction of the preceding saccade. Note that only one conditional is plotted (Figure 8E) because the multimodality of the distribution of saccade directions makes plotting the other conditional in this format inappropriate. Our data suggest that saccades made in upward directions (negative values in Figure 8) are more frequent than saccades in downward directions, and also are followed by shorter duration fixations.

Figure 8B,D,F shows a similar relationship between fixation duration and the direction of the following saccade: upward saccades tend to follow short duration fixations. Again the dependency between direction and fixation duration implies a non-random tendency in oculomotor control, but the nature of this tendency is intriguing. It may be related to the fact that in many natural scenes objects of interest are more likely to be found lower in scenes. If so, we could suggest that saccades directed in an upward direction would likely be followed by fixations in upper portions of the visual scene. Since there will often be little of interest (for example sky in outdoor scenes) in the upper visual field, it might be logical to suggest that fixations are shorter in these uninformative scene regions. It is important to note that we are not here suggesting that it is the visual content of the scene being viewed that gives rise to this tendency (although this is one possible interpretation). Rather we are suggesting that some aspects of systematic tendencies in how we look at scenes may reflect known statistics of the natural environment: We live and operate within a world in which objects of interest are likely to fall in the lower portion of the scene. A systematic tendency to make short fixations after upward saccades could therefore reflect aspects of the natural statistics of the environments in which we operate.

We have already seen that small amplitude saccades are associated with shorter fixations (Figures 5 and 6). Whether upward saccades tend to be of smaller amplitude will be considered in the next section.

Figure 9 shows a strong relationship between fixation duration and the change in direction between the saccades that precede and follow it. As we had suggested from Figure 4, the most frequent occurrence is that the saccade will be in the same direction as the previous, with 180 degree reversals being the second most common occur- 
rence (histogram in lower panel of Figure 9). With regard to fixation duration, we see that fixations are much shorter if the saccades before and after it are in the same direction. There is an approximately linear increase in fixation duration as the magnitude of the change in direction increases. Reversals in saccade direction are associated with the longest fixations. This result could be interpreted in either (or both) of two ways: as a facilitation for saccades in the same direction as the previous, or as an inhibition for saccades in the opposite direction.

A.

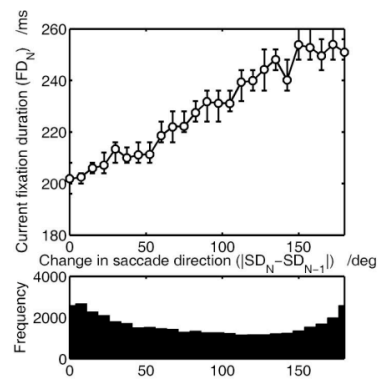

B.

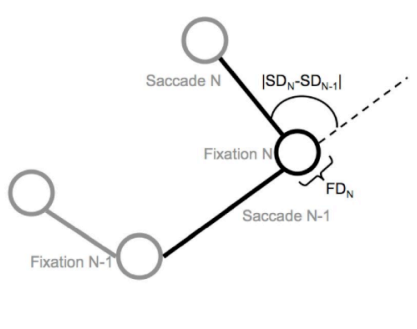

Figure 9. (A) Median current fixation duration (with 95\% confidence intervals) as a function of the absolute change in

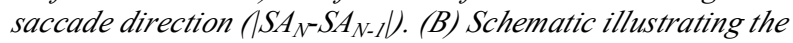
measures plotted in (A).

A facilitation of saccades in the same direction has been reported previously using yellow LEDs as saccade targets, positioned at three degrees to the right or left of a central LED (Carpenter, 2001). Carpenter found that saccades initiated in the same direction as the previous saccade (i.e. that which returned the eye to the central fixation marker LED) were associated with shorter latencies. We therefore extend this result to the situation when viewing complex photographic scenes. One possible explanation for the short duration fixations when continuing in the same direction as the previous saccade is that these are associated with 'corrective' saccades, as we have argued from the sequential dependencies in saccade direction described above (Figure 4). If so, these corrective saccades would be expected to be of smaller amplitude and as we have seen above (Figures 5 and 6) small amplitude saccades tend to be associated with short duration fixations. This of course would predict that a 'corrective' saccade should not only be a saccade in the same direction as the previous, but should also be of small amplitude. Whether this is the case will be explored when we consider the relationship between saccade amplitude and direction in the next section.

Inhibition of return to previously fixated locations is often reported in visual search and tends to be associated with an increase in the duration of the fixation that follows a return to the just-fixated location (Klein \& MacInnes, 1999; Hooge et al., 2005). It is not possible from the present data to distinguish facilitation of continuing in the same direction from inhibition of return, and indeed both mechanisms may contribute to the pattern of data described in Figure 9. It should be noted however, that while the increased fixation duration before saccades back toward a previously-fixated location might imply inhibition of return, the frequency of saccadic reversals does not: Indeed 180-degree reversals are more common than many other changes in direction (Figure 9B, lower panel). This pattern of data is consistent with a previous exploration of inhibition of return by Hooge et al. (2005) who also found both an increase in fixation duration before re-fixating and a high incidence of reversal in saccade direction when viewing complex scenes. For a more detailed consideration of the possible occurrence of inhibition of return in natural scene viewing we refer the reader to Hooge et al.'s paper.

\section{Saccade amplitude and saccade direction}

In this section we explore the relationships between the amplitudes and directions of saccades. This again has received relatively little consideration to date in descriptions of oculomotor behaviour.

Logically, it seems unlikely that there would be any strong relationship between the direction of one saccade and the amplitude of the following saccade. Figure 10A confirms this. However, as can be seen in Figure 10C there is a strong dependency between the direction and amplitude of any given saccade: Saccades in horizontal directions tend to be of much larger amplitude than saccades in vertical or oblique directions. Of course this may result from the aspect ration of the screen: larger saccades are possible in horizontal than vertical directions. That saccade amplitudes might reflect the extent of the visual display has been reported previously (von Wartburg, Pflugshaupt, Wurtz, Nyffeler, Lüthi \& Müri, 2007).

Perhaps more interestingly, saccades in upward directions tend to be of smaller amplitude than saccades in downward directions. This amplitude difference between upward and downward saccades is as we predicted given 
the relationship between saccade direction and fixation duration (Figure 8): Upward saccades are smaller in amplitude and small amplitude saccades tend to be followed by fixations with a short median duration (Figures 5 and $6)$.
A.

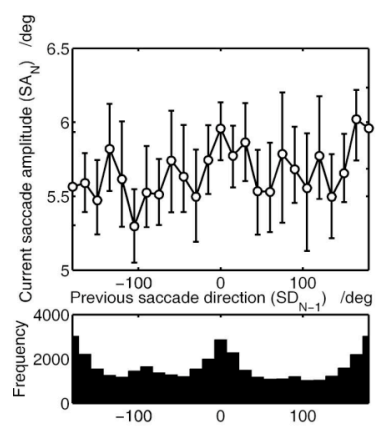

c.

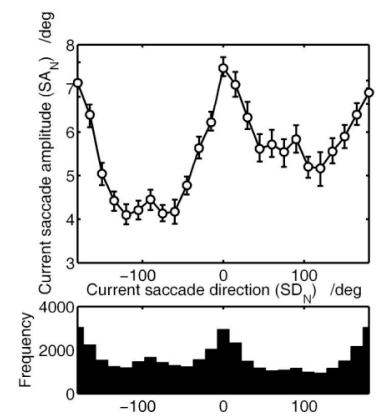

D.

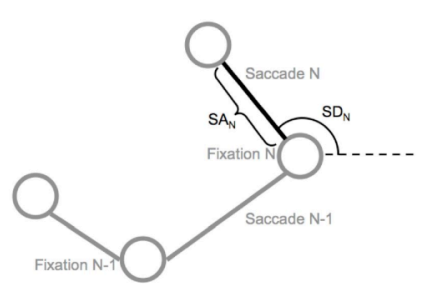

Figure 10. (A,C) Median current saccade amplitude (with 95\% confidence intervals) (A) as a function of previous saccade direction, and (C) as a function of current saccade direction. The histograms below each plot indicate the data that contribute to the above plots. Negative directions indicate upward saccades; positive indicate downward saccades, zero is a saccade to the right, 180 is a saccade to the left. $(B, D)$ Schematics illustrating the measures plotted in (A) and (C).

Figure 11 shows the relationship between the change in direction from previous to current saccade and the amplitude of the current saccade. It is clear that saccades in the same direction (or within around 45 degrees of the same direction) as the previous tend to be of small amplitude. Reversals in direction tend to be associated with large amplitude saccades. This pattern of results is consistent with our prediction based upon the observed relationship between fixation duration and changes in saccade direction (Figure 9). Thus we find that if a saccade continues in the same direction as the previous one it will be of small amplitude and will be preceded by a short duration fixation. Conversely, a reversal in direction will involve a longer fixation pause followed by a larger saccade. This pattern of results is consistent with the possibility we have previously discussed that there is a subpopulation of 'corrective' saccades that are characterised by short fixation pauses, followed by small amplitude saccades in the same direction as the previous, thus correcting what must have been deemed to be a hypometric saccade.

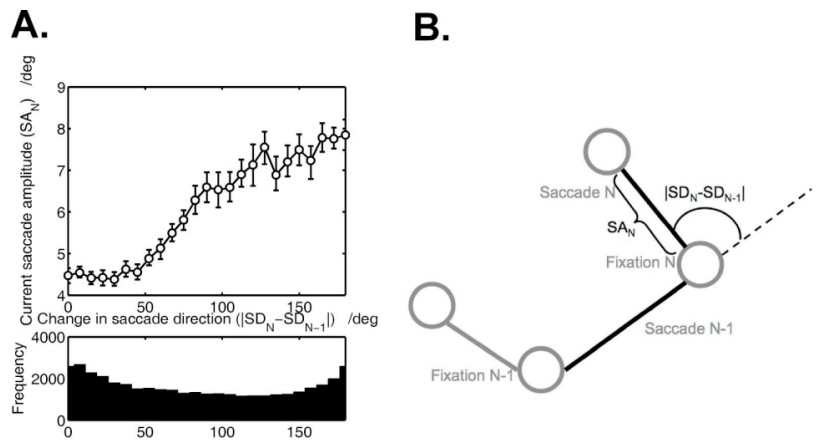

Figure 11. (A) Median current saccade amplitude (with 95\% confidence intervals) as a function of the absolute change in direction between the current and previous saccade. (B) Schematic illustrating the measures plotted in (A).

\section{General Discussion}

When freely viewing images of natural scenes, we find robust relationships between successive saccades and fixations. These relationships reveal a number of insights regarding the manner in which we explore visual scenes. First, we find evidence pertaining to the possibility that we have qualitatively distinct periods of local scanning, separated by larger saccades into new scene regions. Second, we find evidence for the potential existence of 'corrective' saccades when viewing complex scenes. Finally, we can use these findings to argue that these dependencies are highly informative to current models of eye guidance.

\section{Local and global viewing behaviour}

It has previously been argued that there may be two distinct modes of viewing complex scenes: localised or focal inspection, characterised by small amplitude saccades, and global or ambient inspection, characterised by larger amplitude saccades (Frost \& Pöppel, 1976; Pan- 
nasch et al., 2008; Unema et al., 2005). In previous work we considered the relationship between image features selected for fixation and their retinal eccentricity and found that visual features correlated with the targets of small amplitude saccades but not larger amplitude saccades (Tatler et al., 2006). We used this result to support the notion of local and global scanning: local scanning in which visually conspicuous features were selected and global scanning which was not selective of visual features.

In the present paper we find further evidence that supports the qualitative distinction between local scanning and more global relocations in the scene. We find evidence for periods of scanning involving small amplitude saccades and short duration fixations. These periods share the characteristics that would be expected for the local/focal inspection periods that have been suggested by previous researchers.

While we find evidence that is consistent with periods of localised scanning, we do not find evidence to support the suggestion (e.g., by Frost and Pöppel, 1976) that there may be periods during which scanning is more global ${ }^{4}$. Such periods would be expected to be characterised by sequences of large amplitude saccades, yet we find no evidence for this. Instead we find that saccades over 6-7 degrees in extent tend to be followed more frequently by smaller amplitude saccades. As such, our data are consistent with the possibility that periods of localised scanning are separated by single (or few) large amplitude saccades to new regions in the scene, followed by localised scanning of this new region. We also find variation in fixation durations associated with switching between local scanning and global relocation: At the end of a period of local scanning, the final fixation before moving to a new scene region will tend to be short. However, after a large saccade into a new region of the scene, this first fixation, before local scanning begins will tend to be of longer duration, unless the large saccade was deemed to be hypometric (see below).

Our suggested pattern of oculomotor behaviour has interesting parallels with that suggested and described by

\footnotetext{
4

4 However, it should be remembered that our decision not to split our analyses between early and late in the viewing period necessarily excludes our ability to support or deny the existence of an early global period followed by sustained local viewing throughout the remaining presentation time (Unema et al., 2005).
}

Land and colleagues (Land et al., 1999) during natural behaviour. These authors suggested that behaviour could be described as a series of 'Object Related Acts'. In each ORA, vision and action are directed together (usually) to a behaviourally relevant object. Successful completion of the behavioural goal requires the serial completion of a set of ORAs. Within their report it was suggested that during ORAs small amplitude saccades were made, corresponding to fixations within the object or objects required for that specific ORA. At the end of the ORA a large amplitude relocation was made to the target of the next ORA. As such, this describes an oculomotor strategy during this natural behaviour, of making a series of small amplitude saccades, followed by a large amplitude relocation to the next behaviourally relevant target, followed by localised scanning at this new location. This is entirely consistent with the data we have presented in the current report. Of course, at least two factors would result in less pronounced patterns in our dataset. Firstly, presentation of natural images on a computer screen vastly decreases the range of distances between objects compared to that which would occur in natural viewing situations. The maximum size of an exploratory saccade is bounded by the size of the computer screen. Secondly, if we expect a local/global scanning strategy to serve behaviour such as ORAs, then it is reasonable to think that such scanning strategy would be less prominent in our results as compared to a similar experiment with specific viewing instructions or the ability to interact with the scene as in normal viewing.

It should be noted that while we argue for the existence of periods of localised scanning separated by large relocations to new scene regions, we do not suggest that this description encompasses all of the observed fixation behaviour. If this were the case we would expect for example that in Figure 3A, we might see clear bi- (or multi) modality in the relationship between successive saccade amplitudes, revealing distinct modes of scanning. That this is not clear in the Figures suggests that our notion of localised scanning followed by large amplitude relocation to new portions of the scene only describes one aspect of eye movement behaviour while viewing complex scenes.

\section{Corrective saccades in natural scene viewing}

One intriguing and unexpected finding that emerges from our exploration of eye movement behaviour, is the possibility that there may be a subset of saccades that appear to share the characteristics of what would be 
thought of as 'corrective' saccades when viewing simplified stimuli.

When viewing isolated targets in otherwise impoverished visual environments, it is commonly observed that large saccades to these targets tend to be hypometric (i.e. undershoot), and are followed by small amplitude saccades to correct final eye position to being on target (e.g., Becker, 1972, 1991; Carpenter, 1988; Deubel et al., 1982; Kapoula \& Robinson, 1986; Prablanc \& Jeannerod, 1975). The fixation duration between the large hypometric saccade and the small corrective saccade that follows it tends to be very short (Becker, 1972, 1991; Carpenter, 1988). As such, corrective saccades will be a set of saccades that have small amplitudes, continue in the same direction as the previous saccade and are preceded by a short duration fixation.

While there is ample evidence for corrective saccades when viewing isolated targets and simple visual displays, to our knowledge, the extent to which these correction saccades might be a feature of natural viewing behaviour when inspecting images of natural scenes or real environments is uncertain. We know of no study to date that has reported the existence or otherwise of corrective saccades in complex scene viewing. One reason for this is that it is hard to determine what was the intended target of any saccade when there are visual elements throughout the entire visual environment: for example a saccade to a worktop in a kitchen scene might be an inaccurate attempt to target a nearby object or may it may have been that the viewer intended to inspect the work surface. As such, definitive statements about the accuracy of saccades in natural viewing conditions cannot be made; however, inferences and assumptions about the need to correct saccades have been made in previous literature and views about the relative occurrence of corrections differ.

In a recent paper, Hollingworth, Richard \& Luck (2008) argued that because corrective saccades are a common feature of viewing simple visual displays they must therefore be a common feature of viewing complex scene stimuli. With this in mind they investigated the role of visual short term memory across saccades in a viewing paradigm designed to enforce saccade targeting errors that would need to be corrected. These authors concluded that VSTM serves to preserve details of the intended saccade target to allow rapid correction if the saccade is inaccurate.
From studies of eye movements during relatively unconstrained behaviour in real environments, the case for corrective saccades seems less convincing. When manoeuvring an object past an obstacle, it was found that accurate fixation of the obstacle was not required: fixations that landed close to the obstacle but not on it were not corrected (Johansson, Westling, Backstrom \& Flanagan, 2001). Successful completion of the manoeuvre did not rely on correcting saccades that fell short of the obstacle. When making tea, distant objects were found to be targeted accurately by single large amplitude saccades on many occasions (Land et al., 1999). When a single large saccade was not observed, the relocation involved a series of smaller amplitude saccades rather than a large saccade followed by a small correction (Land et al., 1999). Indeed these authors argued that saccades below 2.5 degrees of visual angle were very rare in this natural task. Other studies of natural behaviour also do not present clear arguments for a prevalence of corrective saccades (e.g., Ballard et al., 1992; Hayhoe et al., 2003; Land \& Hayhoe, 2001; Pelz \& Canosa, 2001); certainly not in the order of the $30-40 \%$ of large saccades being hypometric that is reported when viewing simple visual displays (Carpenter, 1988). While evidence from natural behaviour does not argue for a prevalence of hypometric saccades with subsequent correction, the possibility that corrective saccades occur in these situations can by no means be dismissed.

In our data, we find evidence for a subset of saccades that share the characteristics expected for corrective saccades: a set of saccades that continue in the same direction as the previous saccade, have small amplitudes and are preceded by short duration fixations. As such, we suggest that corrective saccades may have been a feature of the eye movement behaviour of our participants as they freely viewed images of natural scenes. To our knowledge this is the first quantitative report of the possible existence of such corrective saccades when viewing complex natural scenes. However, in isolation, these results cannot be extended to suggest that corrective saccades are a feature of natural behaviour. First, viewing a photograph displayed on a computer monitor is not like moving through a real environment. Second, freely viewing scenes with no explicit task instructions does not produce the same visual inspection behaviour as viewing the same scene with a visual task (Yarbus, 1967). Both of these factors might mean that the observed possibility of 
corrective saccades is peculiar to the conditions of our experimental setup.

\section{Systematic tendencies in scene viewing}

It is clear from our explorations of the relationships between the basic oculomotor measures of fixation duration, saccade amplitude and saccade direction, that there are statistical dependencies between these measures. These statistical dependencies can be interpreted as revealing systematic tendencies in the manner in which we move our eyes when inspecting images of complex natural scenes. It is clear that for any fixation or saccade we make, there are reliable influences of the saccades and fixations that immediately precede or follow it. As such, to neglect these systematic tendencies in models of eye movement control, is to neglect a component of oculomotor behaviour that could potentially form an informative part of models of eye guidance.

At present, quantitative models of eye guidance have focussed on the ability to account for fixation selection on the basis of low-level visual features. Arguments against this position have tended to stress the importance of top down factors such as behavioural task, and the inability of feature-based models to account for eye movements under conditions of varying high level task demands (e.g., Henderson et al., 2007; Underwood \& Foulsham, 2006; Underwood et al., 2006). However, to our knowledge no current models attempt to incorporate systematic tendencies in how the eyes are moved, or reliable tendencies between consecutive saccades and fixations, despite evidence for sequential dependencies in eye movements having been described in the literature (e.g. Motter \& Belky, 1998; Unema et al., 2005). Given the present results we argue that a complete understanding of eye guidance when viewing complex natural scenes ought to bear in mind these reliable systematic tendencies in eye movement behaviour.

Consideration of systematic tendencies can help us understand eye guidance in at least two ways. Firstly, considering these tendencies is likely to improve our ability to model and understand eye guidance: initial unpublished work by the authors suggests this is indeed the case. Secondly, knowledge of such systematic tendencies can be used as further empirical benchmark with which to evaluate models of eye guidance. An example of these approaches can be seen with inhibition of return. A 'mechanistic' incorporation of this knowledge was built in to the saliency model by Itti and Koch (2000) by means of a time-decaying inhibitory blob. Incorporating this property into the model obviously resulted in closer matches to human behaviour. Unfortunately this approach does not explain why we exhibit such tendencies. The alternative approach is well demonstrated by Najemnik \& Geisler (2005) who found that inhibition-of-returnlike behaviour simply emerges from their formulation of optimal visual search. Further use of eye movement statistics to evaluate different accounts of eye guidance was recently demonstrated by Najemnik \& Geisler (2008). More detailed description of statistical dependencies between saccades, such as we have outlined, clearly provide more powerful ways to evaluate and compare different models of eye guidance.

\section{Conclusion}

Successive fixations and saccades are not independent events: what the eyes have just done strongly influences the characteristics of each new fixation and saccade. Exploring this finding has highlighted three issues that will be important in our developing understanding of human eye movement behaviour. First, we provide further evidence in support of the existence of qualitatively distinct local scanning and global relocating of gaze (albeit with maybe only a single saccade). Second, we find evidence for the existence of corrective saccades in complex scene viewing. Third, we argue that understanding our observed systematic tendencies in oculomotor behaviour will provide important new insights into our understanding of saccade target selection.

\section{References}

Antes, J. R. (1974). Time Course of Picture Viewing. Journal of Experimental Psychology, 103(1), 62-70.

Baddeley, R. J., \& Tatler, B. W. (2006). High frequency edges (but not contrast) predict where we fixate: a Bayesian system identification analysis. Vision Research, 46, 2824-2833.

Bahill, A. T., Adler, D., \& Stark, L. (1975). Most Naturally Occurring Human Saccades Have Magnitudes of 15 Degrees or Less. Investigative Ophthalmology, 14(6), 468-469. 
Bair, W., \& O'Keefe, L. P. (1998). The influence of fixational eye movements on the response of neurons in area MT of the macaque. Visual Neuroscience, 15(4), 779-786.

Ballard, D. H., Hayhoe, M. M., \& Pelz, J. B. (1995). Memory Representations in Natural Tasks. Journal of Cognitive Neuroscience, 7(1), 66-80.

Ballard, D. H., Hayhoe, M. M., Li, F., Whitehead, S. D., Frisby, J. P., Taylor, J. G., et al. (1992). Hand Eye Coordination During Sequential Tasks. Philosophical Transactions of the Royal Society of London Series BBiological Sciences, 337(1281), 331-339.

Becker, W. (1972). The control of eye movements in the saccadic system. Biblthica ophthal, 82, 233-243.

Becker, W. (1991). Saccades. In R. H. S. Carpenter (Ed.), Vision \& Visual Dysfuntion volume 8: Eye Movements (pp. 95-137). Basingstoke: Macmillan.

Buswell, G. T. (1935). How People Look at Pictures: A Study of the Psychology of Perception in Art. Chicago: University of Chicago Press.

Carpenter, R. H. S. (1988). Movements of the eyes (2nd ed.). London: Pion.

Carpenter, R. H. S. (2001). Express saccades: is bimodality a result of the order of stimulus presentation? $\mathrm{Vi}$ sion Research, 41, 1145-1151.

Deubel, H., Wolf, W., \& Hauske, G. (1984). Corrective saccades: effect of shifting the saccade goal. Vision Research, 22(3), 353-364.

Duncan, J., \& Humphreys, G. W. (1989). Visual search and visual similarity. Psychological Review, 96, 433458.

Duncan, J., \& Humphreys, G. W. (1992). Beyond the visual surface: Visual search and attentional engagement. Journal of Experimental Psychology-Human Perception and Performance, 18, 578-588.

Efron, B., \& Tibshirani, R. J. (1993). An introduction to the bootstrap. New York: Chapman and Hall.

Einhäuser, W., Rutishauser, U., \& Koch, C. (2008). Taskdemands can immediately reverse the effects of sensory-driven saliency in complex visual stimuli. Journal of Vision, 8(2):2, 1-19,

http://journalofvision.org/8/2/2/, doi:10.1167/8.2.2
Frost, D., \& Pöppel, E. (1976). Different Programming Modes of Human Saccadic Eye-Movements as a Function of Stimulus Eccentricity - Indications of a Functional Subdivision of Visual-Field. Biological Cybernetics, 23(1), 39-48.

Gajewski, D. A., Pearson, A. M., Mack, M. L., Bartlett, F. N., \& Henderson, J. M. (2005). Human gaze control in real world search. In Attention and Performance in Computational Vision (Vol. 3368, pp. 83-99).

Hayhoe, M. M., Shrivastava, A., Mruczek, R., \& Pelz, J. B. (2003). Visual memory and motor planning in a natural task. Journal of Vision, 3(1), 49-63.

Henderson, J. M., Brockmole, J. R., Castelhano, M. S., \& Mack, M. L. (2007). Visual saliency does not account for eye movements during search in real-world scenes. In R. P. G. van Gompel, M. H. Fischer, W. S. Murray \& R. L. Hill (Eds.), Eye movements: A window on mind and brain (pp. 537-562). Oxford: Elsevier.

Hollingworth, A., Richard, A. M., \& Luck, S. J. (2008). Understanding the function of visual short-term memory: transsaccadic memory, object correspondence, and gaze correction. Journal of Experimental Psychology-General, 137(1), 163-181.

Hooge, I.T.C., Over, E.A.B., van Wezel, R.J.A. and Frens, M.A. (2005). Inhibition of return is not a foraging facilitator in saccadic search and free viewing, $\mathrm{Vi}$ sion Research, 45, 1901-1908.

Humphrey, K. \& Underwood, G. (2008). Fixation sequences in imagery and recognition during the processing of pictures of real-world scenes. Journal of Eye Movement Research

Ingram, J.N., Körding, K.P., Howard, I.S., and Wolpert, D.M. (2008). The statistics of natural hand movements. Experimental Brain Research, 188(2), 223-236

Itti, L., \& Koch, C. (2000). A saliency-based search mechanism for overt and covert shifts of visual attention. Vision Research, 40(10-12), 1489-1506.

Johansson, R. S., Westling, G. R., Backstrom, A., \& Flanagan, J. R. (2001). Eye-hand coordination in object manipulation. Journal of Neuroscience, 21(17), 6917-6932.

Kapoula, Z., \& Robinson, D. A. (1986). Saccadic undershoot is not inevitable: saccades can be accurate. $\mathrm{Vi}$ sion Research, 26(5), 735-743. 
Klein, R. M., \& MacInnes, W. J. (1999). Inhibition of return is a foraging facilitator in visual search. Psychological Science, 10, 346-352.

Körding, K.P., and Wolpert, D.M. (2004). Bayesian integration in sensorimotor learning. Nature 427, 244247.

Krieger, G., Rentschler, I., Hauske, G., Schill, K., \& Zetzsche, C. (2000). Object and scene analysis by saccadic eye-movements: an investigation with higher-order statistics. Spatial Vision, 13(2-3), 201214.

Land, M. F., \& Hayhoe, M. M. (2001). In what ways do eye movements contribute to everyday activities? $\mathrm{Vi}$ sion Research, 41(25-26), 3559-3565.

Land, M. F., Mennie, N., \& Rusted, J. (1999). The roles of vision and eye movements in the control of activities of daily living. Perception, 28(11), 1311-1328.

Lappe, M., Pekel, M., \& Hoffmann, K. P. (1998). Optokinetic eye movements elicited by radial optic flow in the macaque monkey. Journal of Neurophysiology, 79(3), 1461-1480.

Lee, S. P., Badler, J. B., \& Badler, N. I. (2002). Eyes alive. Acm Transactions on Graphics, 21(3), 637-644.

Mannan, S. K., Ruddock, K. H., \& Wooding, D. S. (1997). Fixation sequences made during visual examination of briefly presented 2D images. Spatial $\mathrm{Vi}$ sion, 11(2), 157-178.

Moeller, G. U., Kayser, C., Knecht, F., \& König, P. (2004). Interactions between eye movement systems in cats and humans. Experimental Brain Research, 157(2), 215-224.

Motter, B. C., \& Belky, E. J. (1998). The guidance of eye movements during active visual search. Vision Research, 38(12), 1805-1815.

Najemnik, J., \& Geisler, W. S. (2005). Optimal eye movement strategies in visual search. Nature, 434 , 387-391.

Najemnik, J., \& Geisler, W. S. (2008). Eye movement statistics in humans are consistent with an optimal search strategy. Journal of Vision, 8(3):4, 1-14,

Navalpakkam, V., \& Itti, L. (2005). Modeling the influence of task on attention. Vision Research, 45(2), 205231.
Nyström, M. \& Holmqvist, K. (2008). Semantic override of low-level features in image viewing - both initially and overall. Journal of Eye Movement Research, 2(2): 2, 1-11.

Pannasch, S., Helmert, J. R., Roth, K., Herbold, A-K., \& Walter, H. (2008). Visual fixation durations and saccade amplitudes: Shifting relationship in a variety of conditions. Journal of Eye Movement Research, 2(2): 4, 1-19.

Parkhurst, D. J., \& Niebur, E. (2003). Scene content selected by active vision. Spatial Vision, 16(2), 125154.

Parkhurst, D. J., Law, K., \& Niebur, E. (2002). Modeling the role of salience in the allocation of overt visual attention. Vision Research, 42(1), 107-123.

Pelz, J. B., \& Canosa, R. (2001). Oculomotor behavior and perceptual strategies in complex tasks. Vision Research, 41(25-26), 3587-3596.

Prablanc, C., \& Jeannerod, M. (1975). Corrective saccades: Dependence of retinal reafferent signals. $\mathrm{Vi}$ sion Research, 15, 465-469.

Reinagel, P., \& Zador, A. M. (1999). Natural scene statistics at the centre of gaze. Network-Computation in Neural Systems, 10(4), 341-350.

Tatler, B. W. (2007). The central fixation bias in scene viewing: selecting an optimal viewing position independently of motor biases and image feature distributions. Journal of Vision, 7(14), 4, 1-17.

Tatler, B. W., Baddeley, R. J., \& Gilchrist, I. D. (2005). Visual correlates of fixation selection: effects of scale and time. Vision Research, 45(5), 643-659.

Tatler, B. W., Baddeley, R. J., \& Vincent, B. T. (2006). The long and the short of it: spatial statistics at fixation vary with saccade amplitude and task. Vision Research, 46, 1857-1862.

Torralba, A., \& Oliva, A. (2003). Statistics of natural image categories. Network-Computation in Neural Systems, 14(3), 391-412.

Torralba, A., Oliva, A., Castelhano, M. S., \& Henderson, J. M. (2006). Contextual guidance of eye movements and attention in real-world scenes: The role of global features in object search. Psychological Review, 113(4), 766-786. 
Treisman, A. M., \& Gelade, G. (1980). A featureintegration theory of attention. Cognitive Psychology, 12, 97-136.

Underwood, G., \& Foulsham, T. (2006). Visual saliency and semantic incongruency influence eye movements when inspecting pictures. Quarterly Journal of Experimental Psychology, 59(11), 1931-1949.

Underwood, G., Foulsham, T., van Loon, E., Humphreys, L., \& Bloyce, J. (2006). Eye movements during scene inspection: A test of the saliency map hypothesis. European Journal of Cognitive Psychology, 18(3), 321-342.

Unema, P. J. A., Pannasch, S., Joos, M., \& Velichkovsky, B. M. (2005). Time course of information processing during scene perception: The relationship between saccade amplitude and fixation duration. Visual Cognition, 12(3), 473-494.

Velichkovsky, B. M., Joos, M., Helmert, J. R., \& Pannasch, S. (2005). Two visual systems and their eye movements: Evidence from static and dynamic scene perception. In B. G. Bara, L. Barsalou \& M. Bucciarelli (Eds.), Proceedings of the XXVII Conference of the Cognitive Science Society (pp. 2283-2288). Mahwah, NJ: Lawrence Erlbaum.

Velichkovsky, B. M., Rothert, A., Kopf, M., Dornhoefer, S. M., \& Joos, M. (2002). Towards an express diagnostics for level of processing and hazard perception. Transportation Research, Part F, 5(2), 145-156.

Velichkovsky, B. M., Rothert, A., Miniotas, D., Dornhoefer, S. M., Joos, M., \& Pannasch, S. (2003). Visual Fixations as a Rapid Indicator of Hazard Perception. In G. H. R. Hockey, A. W. K. Gaillard \& O. Burov (Eds.), Operator functional state and impaired performance in complex work environments (pp. 313321). Amsterdam/Washington: DC: IOS Press (NATO Science Series).

Vincent, B. T., Troscianko, T., \& Gilchrist, I. D. (2007). Investigating a space-variant weighted salience account of visual selection. Vision Research, 47(13), 1809-1820.

Vivani, P., \& Swensson, R. G. (1982). Saccadic eye movements to peripherally discriminated visual targets. Journal of Experimental Psychology-Human Perception and Performance, 8, 113-126.

Viviani, P. (1990). Eye movements in visual search: cognitive, perceptual and motor control aspects. Reviews of Oculomotor Research, 4, 353-393. von Wartburg R, Wurtz P, Pflugshaupt T, Nyffeler T, Lüthi M, Müri R M, (2007). Size matters: Saccades during scene perception. Perception, 36(3), 355-365

Wade, N. J., \& Tatler, B. W. (2005). The Moving Tablet of the Eye: the Origins of modern Eye Movement Research. New York: Oxford University Press.

Wolfe, J. M. (1998). Visual Search. In H. Pashler (Ed.), Attention (pp. 13-74). Hove, East Sussex: Psychology Press.

Yarbus, A. L. (1967). Eye movements and vision. New York: Plenum Press. 